\title{
Selection of phospholipids to design liposome preparations with high skin penetration-enhancing effects
}

Pajaree Sakdiset $^{a, b}$, Akie Okada $^{a}$, Hiroaki Todo $^{a}$, Kenji Sugibayashi $^{a, *}$

5 a Graduate School of Pharmaceutical Sciences, Josai University, 1-1 Keyakidai, Sakado, Saitama 350-0295, Japan,

${ }^{b}$ School of Pharmacy, Walailak University, 222 Thaiburi, Thasala, Nakhon Si Thammarat 80161, Thailand.

$20{ }^{*}$ Corresponding author at: Faculty of Pharmacy and Pharmaceutical Sciences, Josai University, 1-1 Keyakidai, Sakado, Saitama 350-0295, Japan. Tel.: +81-49-271-7367; fax: +81-49-2718137.

E-mail address: sugib@josai.ac.jp (K. Sugibayashi). 
ABSTRACT (not more than 200 words)

The objective of the present study is to search for a good selection method of phospholipids to design liposome preparations with high skin penetration-enhancing effects. Five kinds of phosphatidylcholines and phosphatidylglycerols each were selected. First, phospholipid aqueous dispersions and liposomes containing caffeine as a model drug were

30 tested for their skin penetration-enhancing effects using excised hairless rat skin. As results, 1,2-distearoyl-sn-glycero-3-phosphocholine (DSPC) and 1,2-dipalmitoyl-sn-glycero-3phosphoglycerol, sodium salt (DPPG) dispersions showed high penetration-enhancing ratio $(E R)$, whereas DPPG, 1,2-dilauroyl-sn-glycero-3-phosphocholine (DLPC) and 1,2dimyristoyl-sn-glycero-3-phosphocholine (DMPC) liposomes showed high ER, suggesting that liposomes had different skin penetration-enhancing mechanisms from phospholipid dispersions. Next, two kinds of experiments were done to clarify the possible mechanism of liposomes as follows: the excised skin was pretreated for $1 \mathrm{~h}$ with caffeine-free phospholipid dispersions and liposomes, and caffeine solution was added to determine its skin permeation. Separately, caffeine permeation experiments were done using physical mixture of blank 40 liposomes and caffeine solution (caffeine-spiked liposomes) and caffeine-entrapped liposomes (caffeine was entrapped only in liposomes). As results, DPPG was a promising phospholipid candidate to fabricate liposome formulations with high skin penetration-enhancing effects, since DPPG phospholipid and its liposome vesicles had a combination effect to disrupt the SC lipid barrier as well as could carry both free and entrapped caffeine in the formulation through 45 the skin.

Keywords:

phospholipids, liposomes, skin penetration-enhancing effect, 
50 formulation development

Chemical compounds used in this article:

DLPC (PubChem CID: 512874); DLPG (PubChem CID: 46891823); DMPC (PubChem CID: 5459377); DMPG (PubChem CID: 46891824); DPPC (PubChem CID: 452110); DPPG 55 (PubChem CID: 46891827); DSPC (PubChem CID: 94190); DSPG (PubChem CID: 53487811); DOPC (PubChem CID: 10350317); DOPG (PubChem CID: 23702460); Cholesterol (PubChem CID: 5997); Caffeine (PubChem CID: 2519) 


\section{Introduction}

Liposomes, a type of classical vesicular drug delivery systems, have been received high attention in the field of skin drug delivery due to their ability to entrap drug(s) and enhance the skin penetration of both hydrophilic and lipophilic molecules (Jain et al., 2017; Liu et al., 2004; Yu and Liao, 1996). The main composition of liposomes is an amphiphilic molecule, phospholipids, which can spontaneously form the structure of closed bilayers vesicles as they confront with water (Jadhav et al., 2012). The mechanisms to enhance the skin penetration of drugs by liposomes have been proposed including (1) free drug operation, (2) intact vesicular penetration, (3) vesicle adsorption to and/or fusion with the stratum corneum (SC) and (4) their penetration-enhancing effect (El Maghraby et al., 2006). However, conflicting results on these mechanisms have been found in spite of much efforts made by many researchers.

One of the well-accepted mechanisms for the skin penetration-enhancement by liposomes is the penetration of the amphiphilic components into the skin barrier and their perturbation actions on the packing of SC lipids (Kato et al., 1987; Kirjavainen et al., 1996). From this reason, liposome composition must be an important parameter for the enhancing effect of liposomes on the skin penetration of drugs. In the formulation design of liposomes, many researches have already focused on the optimization of liposomal characteristics; i.e., morphology, vesicular size, surface charge, entrapment efficiency, transition temperature or elasticity of liposomes by changing the liposome compositions or their preparation procedure (Gillet et al., 2011a, 2011b; Verma et al., 2003a). Moreover, novel classes of vesicular carriers have been developed to obtain the high skin penetration-enhancing effect of liposomes, by addition of edge activators or chemical penetration enhancers into the classical liposomes (Duangjit et al., 2011; Gillet et al., 2011a; Manconi et al., 2011; Touitou et al., 2000). However, the skin penetration-enhancing effects of the main phospholipid compositions in liposomes were not fully clarified yet. 

penetration-enhancing effects of a model hydrophilic drug, caffeine, through excised hairless rat skin using a currently established assay system comprised of multiple-diffusion cells as a screening approach, since phospholipids as the liposome composition can play an important role in the skin penetration-enhancing effect of liposomes. Then, several kinds of phospholipids were selected to prepare liposomes and tested for their skin penetration-enhancing effects, and the obtained results were compared to those for their phospholipid dispersions to design suitable liposomes having high skin penetration-enhancing effects. Next, two kinds of further permeation experiments were done in order to clarify their possible modes of action of each phospholipid for their skin penetration-enhancing effects as follows: The effect of 1 hpretreatment on the excised skin was evaluated with caffeine-free phospholipid dispersions and liposomes. Caffeine solution was added after the pretreatment to carry out the general skin permeation experiment. Separately, caffeine permeation experiments were done using physical mixture of blank liposomes and caffeine solution (caffeine-spiked liposomes) and caffeineentrapped liposomes (caffeine was entrapped only inside of liposomes). skin penetration-enhancing effect of a model hydrophilic drug, caffeine.

\section{Materials and methods}

\subsection{Materials}

Phospholipids including 1,2-dilauroyl-sn-glycero-3-phosphocholine (abbreviated as in DLPC; the same as below), 1,2-dimyristoyl-sn-glycero-3-phosphocholine (DMPC), 1,2dimyristoyl-sn-glycero-3-phospho-glycerol, sodium salt (DMPG), 1,2-dipalmitoyl-sn-glycero3-phosphocholine (DPPC), 1,2-dipalmitoyl-sn-glycero-3-phosphoglycerol, sodium salt (DPPG), 1,2-distearoyl-sn-glycero-3-phosphocholine (DSPC), 1,2-distearoyl-sn-glycero-3- 
phosphoglycerol, sodium salt (DSPG), 1,2-dioleoyl-sn-glycero-3-phosphocholine (DOPC) and 1,2-dioleoyl-sn-glycero-3-phosphoglycerol, sodium salt (DOPG) were purchased from NOF Corporation (Tokyo, Japan). 1,2-Dilauroyl-sn-glycero-3-phosphoglycerol, sodium salt (DLPG) was obtained from Olbracht Serdary Research Laboratories (Toronto, ON, Canada). Table 1 summarizes the abbreviations and number of carbon atom and double bond in the alkyl chain 115 for the phopholipids .

Table 1

Cholesterol was purchased from Sigma-Aldrich (St. Louis, MO, U.S.A.). Caffeine, chloroform, methanol and ethanol were purchased from Wako Pure Chemicals Industries, Ltd. (Osaka, Japan). These reagents were used without further purification.

\subsection{Experimental animals}

Male WBN/ILA-Ht hairless rats, weighing between 200 and 260 g, were obtained

125 from the Life Science Research Center, Josai University (Sakado, Saitama, Japan) and Ishikawa Experimental Animal Laboratories (Saitama, Japan). Rats were bred in a room maintained at $25 \pm 2^{\circ} \mathrm{C}$, in which the on and off times for the lighting were 07:00 and 19:00, respectively. Animal had free access to water and food (MF, Oriental Yeast Co., Ltd., Tokyo, Japan).

All breeding procedures and experiments on the animals were performed in accordance with the guidelines of the Animal Experiment Committee of Josai University.

The abdominal skin from hairless rats was excised under anesthesia by i.p. injection of anesthesia containing medetomidine $(0.375 \mathrm{mg} / \mathrm{kg})$, butorpharnol $(2.5 \mathrm{mg} / \mathrm{kg})$ and 
midazolam (2 mg/kg). After that, the hairless rats were sacrificed immediately by injection of pentobarbital sodium (40 mg/kg).

\subsection{Preparation of liposomes}

Liposomes were prepared using phospholipids and cholesterol in a ratio of 4:1 w/w. The compositions were dissolved in chloroform: methanol $(2: 1 \mathrm{v} / \mathrm{v})$ in a round-bottomed flask and the solvent was evaporated to form the thin film using a rotary evaporator under reduced pressure. The obtained film was purged with nitrogen gas and kept overnight to remove the trace organic solvent. After that, the flask was immersed in a water bath at $90^{\circ} \mathrm{C}$ for annealing of the thin film for $30 \mathrm{~min}$, and then $100 \mathrm{mM}$ caffeine in phosphate buffered saline $\mathrm{pH} 7.4$ (PBS) solution was added to adjust phospholipid concentration to 3\% (w/v). The thin film was hydrated for 30 min and the resulting liposomes containing caffeine were then sonicated using a probe sonicator (VCX-750, Sonics \& Materials Inc., Newtown, CT, USA) for 30 s. Next, 4 cycles of freeze-thaw process were performed by immersing the flask in liquid nitrogen and in $90^{\circ} \mathrm{C}$-water bath for 3 min each. The obtained liposomes were further extruded using a miniextruder (Avanti Polar Lipids, Inc., Alabaster, AL, USA) assembled with a membrane filter (with pore sizes of 400, 200 and $100 \mathrm{~nm}$, Whatman ${ }^{\circledR}$ track-etched membranes, GE Healthcare Japan, Tokyo, Japan). All final liposome formulations containing caffeine were kept at $25^{\circ} \mathrm{C}$ and freshly used for the skin permeation experiment within the next day after preparation. In the final formulations, caffeine must be contained both in the inside and outside of liposomes. These liposome formulations were used to evaluate the caffeine permeation through skin. without addition of caffeine. 


\subsection{Characterizations of liposomes}

\subsubsection{Particle size and zeta potential}

The particle size and zeta potential of liposomes were measured after 100-fold dilution with PBS using a Zetasizer Nano ZS (Malvern Instruments Ltd., Malvern, UK). The size measurements were performed at $25^{\circ} \mathrm{C}$ and a scattering angle of $90^{\circ}$. Individual zeta potential measurement was repeated for at least 10 readings from each liposome sample.

\subsubsection{Caffeine distribution in liposome formulations}

The entrapment efficiency ( $E E$ ) of caffeine in each liposome sample was determined by ultracentrifuge technique to evaluate the caffeine distribution in either inside and outside of liposome formulations. Final liposome suspension $(400 \mu \mathrm{L})$ was put in a centrifuge tube and centrifuged using a micro-ultracentrifuge (Himac CS120GXII, Hitachi Koki Co., Ltd., Tokyo, Japan) at $289,000 \times \mathrm{g}, 4^{\circ} \mathrm{C}$ for 20 min to separate the liposome pellet (entrapped drug, $E_{d r u g}$ ) from the supernatant (unentrapped drug, $U_{d r u g}$ ). The supernatant was collected and the free caffeine content was determined after 10 -fold dilution with ethanol followed by 10 -fold with PBS. In addition, the entrapped drug content in the inside of liposomes was determined by dispersing the packed liposome pellet with $400 \mu \mathrm{L}$ PBS and further disrupting with 10-fold ethanol followed by dilution with 10-fold PBS. Caffeine contents were analyzed by an HPLC and the \% EE was calculated according to the following equation.

$$
\% \text { Entrapment efficiency }(E E)=\left(\frac{E_{d r u g}}{E_{d r u g}+U_{d r u g}}\right) \times 100
$$




\subsubsection{Differential Scanning Calorimetry}

The phase transition temperature of liposomes $\left(T_{m}\right)$ was determined by a differential scanning calorimeter (DSC) (Thermo plus EVO/ DSC8230, Rigaku Corporation, Akishima, Tokyo, Japan). About $5 \mathrm{mg}$ of liposome pellets obtained from the ultracentrifugation process as described in section 2.4.2, were placed in an aluminum pan. An empty pan was used as a reference. The DSC heating scan was performed at a heating rate of $5.0^{\circ} \mathrm{C} / \mathrm{min}$ in a $15-80^{\circ} \mathrm{C}$ range.

\subsection{In vitro skin permeation experiment}

2.5.1 Determination of cumulative amount of caffeine permeated through skin over $12 \mathrm{~h}$ from different phospholipid dispersions

First, the skin penetration-enhancing effect of different types of phospholipids were determined using a currently designed diffusion cell array system as shown in Fig. 1 (Ikeda Scientific Co., Ltd., Tokyo, Japan). This system is comprised of 12 wells wherein the donor compartments are above the receiver chambers with the excised skin sandwiched between them. This system can simultanously determine 12 sets of permeation data at a single run of the experiment. However, the time course of the cumulative amount of caffeine that permeated through skin could not be determined using this system which differs from Franz-type diffusion cell because of lack of sampling port. Effective permeation area and receiver volume for each well are $0.785 \mathrm{~cm}^{2}$ and $1.36 \mathrm{~mL}$, respectively. The study was performed using excised abdominal skin from hairless rat after removing subcutaneous fat. The skin was excised and cut into two pieces of $3 \times 4 \mathrm{~cm}$ size from the middle line of rat abdomen and set on the diffusion cell array system. At first, 1.0 and 1.36 mL PBS were added in each donor and receiver chamber, respectively, for $1 \mathrm{~h}$ for skin hydration. After that, PBS was removed from donor compartment and replaced with $200 \mu \mathrm{L}$ of 3\%(w/v) phospholipid dispersions containing caffeine in PBS at 
a concentration of $100 \mathrm{mM}$. The permeation experiment was performed at $32^{\circ} \mathrm{C}$ using a thermoshaker at the rotation speed of $200 \mathrm{rpm}$ and the receiver solution was stirred using a stir ball determine the cumulative amount of caffeine that permeated per unit area of skin $\left(Q_{12}\right)$ by an HPLC. Caffeine solution (100 mM) in PBS was used as a control and calculated for skin penetration-enhancement ratio $(E R)$ of each sample by the following equation;

$$
E R=\left(\frac{Q_{12, \text { sample }}}{Q_{12, \text { control }}}\right)
$$

215

where $Q_{12}$, sample and $Q_{12}$, control are the cumulative amount of caffeine permeated per unit area of skin over $12 \mathrm{~h}$ from different phospholipid dispersions and control solution, respectively.

Fig. 1

220

2.5.2 Determination of time course of skin permeation of caffeine from different liposome formulations

Excised abdominal skin from hairless rat was mounted in a vertical-type Franz diffusion cell (effective permeation area of $1.77 \mathrm{~cm}^{2}$ and receiver cell volume of $6.0 \mathrm{~mL}$ ) with the SC side facing the donor cell and the dermal side facing the receiver cell. The receiver and donor cells were filled with 6.0 and $1.0 \mathrm{~mL}$ of PBS, respectively, for $1 \mathrm{~h}$ for skin hydration. Then, $400 \mu \mathrm{L}$ of liposomes containing caffeine at a concentration of $100 \mathrm{mM}$ were replaced in the donor compartment to determine its skin permeation at $32^{\circ} \mathrm{C}$ over $8 \mathrm{~h}$, while the receiver solution was agitated at $500 \mathrm{rpm}$ using a magnetic stirrer. At predetermined times, $0.5 \mathrm{~mL}$ aliquot were collected and the same volume of PBS was added to keep the volume constant. The amount of caffeine permeated through skin was determined by an HPLC. 
2.5.3 Determination of the effect of pretreatment of caffeine-free phospholipid dispersions and liposomes on the caffeine permeation through skin

Selected caffeine-free liposomes $(400 \mu \mathrm{L}$ ) prepared using phospholipids (DPPG, DLPC or DSPG) were applied onto the SC surface of skin for $1 \mathrm{~h}$ after the hydration period with PBS. Phospholipid dispersions (3\% DPPG, DLPC or DSPG) without caffeine were also applied on skin for comparison. Then, the liposomes or phospholipid dispersion without caffeine were removed from the skin surface by washing with $1.0 \mathrm{~mL}$ fresh PBS for 10 times. Caffeine solution $(100 \mathrm{mM}, 400 \mu \mathrm{L})$ was then applied on the skin. The skin permeation experiment was conducted using vertical-type Franz diffusion cell for $8 \mathrm{~h}$ as described in section 2.5.2.

2.5.4 Determination of the skin permeation of caffeine from the physical mixture of blank liposomes and caffeine solution (caffeine-spiked liposomes) prepared by mixing the same volume of the double-concentrated liposomes (preparation method was similar to the final liposomes as above) and $200 \mathrm{mM}$ caffeine. The resultant caffeine-spiked liposomes (400 $\mu \mathrm{L}$ ) were used for the skin permeation experiment of caffeine using vertical-type Franz diffusion cell.

\subsubsection{Determination of the skin permeation of caffeine from caffeine-entrapped liposomes}

Caffeine-entrapped DPPG, DLPC and DSPG liposomes were obtained by ultracentrifugation separation as described in section 2.4.2. In the preparation process, free caffeine was totally removed and the remained caffeine-entrapped liposomes pellet was dispersed with PBS (400 $\mu \mathrm{L})$. The skin permeation experiment using this caffeine-entrapped liposome formulation was performed and compared with the same concentration of free caffeine solution using vertical-type Franz diffusion cell. 


\subsection{Determination of caffeine concentration}

Concentration of caffeine was determined using an HPLC system (Prominence, Shimadzu Corporation, Kyoto, Japan) equipped with a UV detector (SPD-M20A, Shimadzu Corporation). The sample solutions were mixed with the same volume of methanol and then centrifuged at $21,500 \times g$ and $4^{\circ} \mathrm{C}$ for $5 \mathrm{~min}$. The supernatant $(20 \mu \mathrm{L})$ was injected directly into the HPLC system. Chromatographic separation was performed at $40^{\circ} \mathrm{C}$ using an Inertsil ODS3 (5 $\mu \mathrm{m}$ in diameter) entrapped in a column (4.6 mm I.D. x $150 \mathrm{~mm}$, GL Sciences Inc., Tokyo, Japan). The mobile phase was $0.1 \%$ phosphoric acid : methanol $(7: 3 \mathrm{v} / \mathrm{v})$ and the flow rate was $1.0 \mathrm{~mL} / \mathrm{min}$. The detection was performed at UV $280 \mathrm{~nm}$.

\subsection{Statistical analysis}

Data were expressed as the mean \pm S.E. or S.D. The differences among the obtained data were analyzed using unpaired $t$-test. The differences were considered to be significant when $p<0.05$.

\section{Results}

\subsection{Characteristics of liposomes}

Table 2 summarizes the physicochemical properties of liposomes prepared in this study. All liposome formulations had a small diameter in a range of 110-185 nm. Phosphatidylcholine (DLPC, DMPC, DPPC, DSPC and DOPC) liposomes showed larger particle size than phosphatidylglycerol (DLPG, DMPG, DPPG, DSPG and DOPG) liposomes. The zeta potential of these phosphatidylcholine liposomes had quite neutral charge, whereas phosphatidylglycerol liposomes had negative surface charge less than $-40 \mathrm{mV}$. The $E E$ was less than $50 \%$ and $T_{m}$ was within a range of $41-66^{\circ} \mathrm{C}$ for all liposomes. 


\subsection{Skin penetration-enhancing effect of phospholipid dispersions}

Figure 2 shows the obtained $E R$ of hairless rat skin permeation of caffeine in the presence of 3\% phospholipid dispersion in PBS against that of free caffeine solution in PBS (without phospholipids). Currently designed diffusion cell array system (Fig. 1) was used in this experiment to determine $Q_{12}$. $Q_{12}$ was used as an index for skin permeation of caffeine, since data over $12 \mathrm{~h}$ was more reliable to the shorter periods like $8 \mathrm{~h}$ (data not shown). Both DSPC and DPPG dispersions significantly enhanced the skin permeation of caffeine (ER was 1.93 and 1.57, respectively), whereas DOPG dispersion showed significantly lower skin permeation than the control $(\mathrm{PBS})(E R=0.38)$. The other phospholipid dispersions showed almost the same permeation of caffeine to the control (PBS).

\subsection{Skin permeation of caffeine from liposomes}

Then, liposomes prepared from different phospholipids were determined for their skin penetration-enhancing of caffeine. Franz diffusion cell sets were used in this experiment. The experimental period using Franz cells was decided to 8 h, since the reliablity was proven due to the time course data. Figure 3 shows the time course of the cumulative amount of caffeine that permeated through skin from different kinds of liposomes over 8 h. Typical lag time and following steady-state permeation profiles were observed for all liposome preparations prepared in this experiment. Only DPPG, DLPC and DMPC liposomes significantly promoted the caffeine permeation compared to control (caffeine solution in PBS) (ER was 5.43, 3.17 and 2.17, respectively), whereas DPPC and DOPG liposomes significantly decreased the caffeine permeation ( $E R$ was 0.21 and 0.27 , respectively). No or little change in the skin 
penetration was found by the other phospholipid liposomes.

Then, the skin penetration-enhancing effect by phospholipid dispersions and liposome preparations were summarized to compare them as shown in Fig. 4. Interestingly, DLPC, DMPC and DPPG liposomes showed significant higher ER compared to their phospholipid dispersions, while DPPC and DSPC liposomes showed significant lower ER compared to their phospholipid dispersions.

Fig. 4

Although DSPC phospholipid provided the highest ER in the form of dispersion, the skin permeation of caffeine from DSPC liposomes was substantially decreased. DPPG showed the enhancement effect both by phospholipid dispersions and liposomes. However, its $E R$ was highly increased by modification to the liposome formulation. In case of other phospholipids such as DLPG, DMPG, DSPG, DOPC and DOPG, their liposomes showed no significant difference in the $E R$ to those from their dispersions.

Especially in case of DPPG, liposome formulations showed markedly high skin penetration-enhancing effects, although the mechanism was not clarified yet. Then, the following experiments were designed. 


\subsection{Effect of pretreatment with caffeine-free phospholipid dispersions and liposomes on the skin permeation of caffeine}

DPPG and DLPC liposomes exhibited the highest skin penetration-enhancement effects among all liposomes, but DSPG liposomes exhibited low skin permeation of caffeine. Then these three phospholipids were selected and 1 h-pretreatment experiment using caffeinefree phospholipid dispersions or liposomes was done before skin permeation measurement from caffeine solution to clarify the possible skin penetration-enhancing effect of liposomes. Figure 5 shows the results. The pretreatment with phospholipid dispersions enhanced caffeine permeation for DLPC $(E R=1.35)$ and DPPG $(E R=2.47)$, but decreased for DSPG $(E R=0.48)$. The pretreatment experiment was also performed using caffeine free-liposomes. Interestingly, the 1 h-pretreatment with caffeine free-liposomes showed different results: the lower $E R$ was observed compared to the effect of pretreatment with phospholipids dispersions. The ER for caffeine free-DLPC, DPPG and blank DSPG liposomes were 0.87, 1.50 and 0.46, respectively.

\subsection{Effect of physical mixture of blank liposomes and caffeine solution (caffeine-spiked liposomes) on the skin permeation of caffeine}

In order to evaluate the contribution of caffeine contents in the inside and outside of liposomes on its skin permeation, physical mixture of blank liposomes and caffeine was applied on the excised skin to measure the skin permeation of caffeine. The results are shown in Fig. 6. Caffeine-spiked DLPC and DSPG liposomes exhibited lower skin permeation of caffeine ( $E R$ was 0.58 and 0.44 , respectively). On the other hand, only the caffeine-spiked DPPG liposomes significantly enhanced the skin permeation of caffeine $(E R=2.65)$ compared to control caffeine solution. 
Fig. 6

\subsection{Effect of caffeine-entrapped liposomes on the skin permeation of caffeine}

Next, caffeine entrapped liposomes were evaluated for the skin permeation of caffeine.

Figure 7 shows the results. Caffeine-entrapped DPPG liposomes showed the highest caffeine permeation $(E R=4.39)$, whereas caffeine-entrapped DLPC liposomes enhanced about 1.65fold compared to caffeine solution which contained the same concentration as in liposome formulations. No penetration enhancing effect was observed for the caffeine-entrapped DSPG liposomes.

Fig. 7

\section{Discussion}

Although several studies have reported on the potential of liposomes as a topical/transdermal drug delivery system compared to conventional formulations (Foldvari, 1994; Michel et al., 1992; Yu and Liao, 1996), there seems to be a general lack of understanding among researchers regarding on the formulation factors of liposomes to provide high skin penetration-enhancing effects. Therefore, the present study was mainly focused on the designing strategies of liposomes by investigating the effect of phospholipid composition of liposomes on the enhanced skin permeation of drugs.

First, the skin penetration-enhancing effect of different phospholipids used for liposome preparations was evaluated using caffeine as a model penetrant, since the selection of phospholipids must be very important to design suitable liposomes formulations. Among 10 kinds of phospholipids (5 kinds each of phosphatidylcholines and phosphatidylglycerols), 
DPPG and DSPC dispersions significantly improved the skin permeation of caffeine, whereas DOPG dispersion markedly decreased the skin permeation. In addition, the other phospholipids had no or little skin penetration-enhancing effects against the control group (Fig. 2).

Limited studies have reported the skin penetration-enhancing effect of phospholipids (Junyaprasert et al., 2013; Yokomizo, 1996; Yokomizo and Sagitani, 1996). It is a pity that propylene glycol was used to dissolve the phospholipids, which differ from the present study (We used PBS). The present results showed that all phospholipids provided only mild penetration-enhancing effect or decreased the skin permeation of caffeine. Valjakka-Koskela et al. (1998) reported that phospholipids inhibited the skin permeation of naproxen from aqueous gel, but only the gel containing ethanol or propylene glycol as a co-solvent increased the skin permeation of the drug. In addition, Yokomizo and Sagitani (1996) reported that the penetration-enhancing effect of phospholipids was affected by their solubility in solvents. Thus, organic solvents like propylene glycol may overestimate the effect of phospholipids on drug permeation (El Maghraby et al., 2000)

Although the size of liposomes prepared in the present study was equally small (110$180 \mathrm{~nm}$ ), but $T_{m}, E E$ and zeta potential were dramatically affected by changes of the kinds of phospholipids (Table 2). The reason for the differences in the skin penetration-enhancing effects depending on the phospholipids is still unknown with these parameters.

The reason why only DPPG, DLPC and DMPC liposomes showed higher permeation 400 than other liposomes (Fig. 3) can be explained as follows: (1) Some liposomes might have a rigid structure that could form an extra lipid barrier on the skin surface which retards the skin permeation of caffeine, and this extent must be different depending on the phospholipids, or (2) some liposomes might release caffeine in a slower rate than other phospholipid-based liposomes probably due to the interaction between the drug and phospholipids (Kirjavainen et 
The penetration-enhancement ratios $(E R)$ by the phospholipid dispersions were different from those by their liposome formulations (Fig. 4), suggesting that liposome containing caffeine could have different mechanisms to increase the skin permeation than the phospholipid dispersions.

Understanding of different physiochemical properties of phospholipids and liposomes may be necessary to clarify the skin penetration-enhancing mechanism of phospholipids and liposomes. DLPC, DPPG, and DSPG were selected, because DLPC showed a high ER only in the dispersion form and DPPG showed a high effect in the both forms, whereas DLPC had no effect in the both forms. For the above purposes, the skin pretreatment approach and following 415 skin permeation experiments of caffeine were carried out using caffeine-free phospholipids dispersions and liposomes (Fig. 5). In addition, the effect of physical mixture of blank liposomes and caffeine solution (caffeine-spiked liposomes) and caffeine-entrapped liposomes (caffeine presented only in the liposomes) were determined (Figs. 6 and 7, respectively). Pretreatment with DLPC and DPPG dispersions enhanced the skin permeation of caffeine (Fig.

420 5), indicating that these phospholipids had skin penetration-enhancing effects, since they might rearrange and fuse with an ordered structure of intercellular lipids (like ceramides) to reduce the SC barrier function made by disruption of well-packed intercellular lipids and creation of a permeation pathway for drugs (Kato et al., 1987; Mahrhauser et al., 2015; Zellmer et al., 1995). Interestingly, the pretreatment of caffeine-free liposomes provided lower $E R$ than their 425 corresponding phospholipid dispersions (Fig. 5). This could be due to the presence of such vesicles having less fluidity to disrupt the rigid structure of SC than its dispersion forms. Obviously, only the pretreatment with caffeine-free DPPG liposomes increased the skin permeation of caffeine.

For the physical mixture of blank liposomes and caffeine, caffeine-spiked DPPG 430 liposomes could deliver the drug through skin with the highest ER (Fig. 6). Caffeine-free DPPG 
liposomes might disrupt the SC structure, allowing the free caffeine being mixed outside the liposome vesicles to diffuse through the skin barrier. In consequence, the caffeine-entrapped DPPG liposomes also provided the highest ER compared to the caffeine-entrapped DLPC and DSPG liposomes (Fig. 7).

The highest skin penetration-enhancing effect observed from DPPG liposomes was thus due to the synergistic of different actions; the skin penetration-enhancing effect of DPPG dispersions and the skin penetration-enhancing effect of caffeine-free DPPG liposomes as well as the penetration-enhancing ability of caffeine both outside (caffeine-spiked liposome) and inside (caffeine-entrapped liposome) of liposomal vesicles. As similar to the previous report

440 (Verma et al., 2003b), the penetration of non-entrapped and entrapped hydrophilic fluorescence probe, carboxyfluorescein, in liposomes through human skin were increased compared to control solution. The fluorescent may be penetrated along with intact liposomes or associated with liposomal fragment.

The skin penetration-enhancing effect of DLPC liposomes was observed after the 445 pretreatment only with its phospholipid dispersions. Furthermore, only caffeine-entrapped DLPC liposomes showed enhance caffeine permeation. Thus, the overall skin penetrationenhancing effect obtained from DLPC liposomes were lower compared to DPPG liposomes. On the other hand, no skin penetration-enhancing effect was observed for DSPG liposomes in all cases resulting in low caffeine permeation.

Lipophilic tails of fatty acids and phospholipids are known to increase the skin permeation of drugs. Kim et al. (2008) have investigated the effect of carbon-chain length of saturated fatty acids on the skin penetration-enhancing effect and revealed a parabolic correlation between the penetration-enhancing effect and their carbon-chain length of the saturated fatty acids These results suggested that fatty acids with a certain-chain length possess an optimal partition coefficient or solubility parameter. As the carbon-chain length of lipophilic 
tails in phospholipid increased from C12 (DLPC) to C18, (DSPC) in the present experiment, the skin permeation was increased in the dispersion form. Since interaction between liposome membrane and SC intercellular lipids was not investigated yet, the reason for the highest skin permeation from DLPC liposomes is still unclear.

The modes of action of liposomes to enhance the skin permeation of drugs observed in the present study were summarized as follows; (1) the phospholipid molecule could disrupt the SC lipid barrier and enhance the drug permeation, (2) The free liposome vesicles themselves could also disrupt the SC lipid, and (3) drug could associate outside or encapsulate inside of liposome vesicles and then liposomes carry the drug to pass through the skin. However, different compositions of liposomes resulted in different degree of those effects. Further studies should be carried out to understand the molecular mechanisms of each liposomes composition on their skin penetration-enhancing effect.

\section{Conclusion}

Our findings exhibited that composition of liposomes must be an important factor to improve their performance. Understanding the effect of such factors of liposomes could enable researchers to develop the effective liposome formulation with high skin permeation of drugs.

\section{Conflict of interest}

The authors declare no conflict of interest. 


\section{References}

Duangjit, S., Opanasopit, P., Rojanarata, T., Ngawhirunpa, T., 2011. Characterization and in vitro skin permeation of meloxicam-loaded liposomes versus transfersomes. J. Drug Deliv. 9 pages. DOI: $\underline{10.1155 / 2011 / 418316}$

El Maghraby, G.M.M., Williams, A.C., Barry, B.W., 2000. Skin delivery of oestradiol from lipids vesicles: important of liposome structure. Int. J. Pharm. 204, 159169. https://doi.org/10.1016/S0378-5173(00)00493-2

El Maghraby, G.M., Williams, A.C., Barry, B.W., 2006. Can drug-bearing liposomes penetrate intact skin. J. Pharm. Pharmacol. 58, 415-429. DOI: 10.1211/jpp.58.4.0001

Foldvari, M., 1994. In vitro cutaneous and percutaneous delivery and in vivo efficacy of tetracaine from liposomal and conventional vehicles. Pharm. Res. 11, 1593-1598. PMID: 7870676

Gillet, A., Lecomte, F., Hubert, P., Ducat, E., Evrard, B., Piel, G., 2011a. Skin penetration behaviour of liposomes as a function of their composition. Eur. J. Pharm. Biopharm. 79, 43-53. DOI: 10.1016/j.ejpb.2011.01.011

Gillet, A., Compere, P., Lecomte, F., Hubert, P., Ducat, E., Evrard, B., Piel, G., 2011b. Liposome surface charge influence on skin penetration behaviour. Int. J. Pharm. 411, 223231. https://doi.org/10.1016/j.ijpharm.2011.03.049

Jadhav, S.M., Morey, P., Karpe, M., Kadam, V., 2012. Novel vesicular system: an overview. J. Appl. Pharm. Sci. 2, 193-202.

Jain, S., Patel, N., Shah, M.K., Khatri, P., Vora, N., 2017. Recent advances in lipid-based vesicles and particulate carriers for topical and transdermal application. J. Pharm. Sci. 106, 423-445. DOI: 10.1016/j.xphs.2016.10.001

500 Junyaprasert, V.B., Singhsa, P., Jintapattanakit, A., 2013. Influence of chemical penetration enhancers on skin permeability of ellagic acid-loaded niosomes. Asian J. Pharm. Sci. 8, 
110-117. https://doi.org/10.1016/j.ajps.2013.07.014

Kato, A., Ishibashi, Y., Miyake, Y., 1987. Effect of egg yolk lecithin on transdermal delivery of bunazosin hydrochloride. J. Pharm. Pharmacol. 39, 399-400. PMID: 2886592

Kim, M.J., Doh, H.J., Choi, M.K., Chung, S.J., Shim, C.K., Kim, D.D., Kim, J.S., Yong, C.S., Choi, H.G., 2008. Skin permeation enhancement of diclofenac by fatty acids. Drug Deliv. 15, 373-379. DOI: $\underline{10.1080 / 10717540802006898}$

Kirjavainen, M., Urtti, A., Jaaskelainen, I., Suhonen, T.M., Paronen, P., Valjakka-Koskela, R., Kiesvaara, J., Monkkonen, J., 1996. Interaction of liposomes with human skin in vitro the influence of lipid composition and structure. Biochim. Biophys. Acta. 1304, 179-189. https://doi.org/10.1016/S0005-2760(96)00126-9

Kirjavainen, M., Urtti, A., Valjakka-Koskela, R., Kiesvaara, J., Monkkonen, J., 1999.

Liposome-skin interactions and their effects on the skin permeation of drugs. Eur. J. Pharm. Sci. 7, 279-286. https://doi.org/10.1016/S0928-0987(98)00037-2

515 Liu, H., Pan, W.S., Tang, R., Luo, S.D., 2004. Topical delivery of different acyclovir palmitate liposome formulations through rat skin in vitro. Pharmazie. 59, 203-206. PMID: 15074594 Mahrhauser, D.S., Reznicek, G., Gehrig, S., Geyer, A., Ogris, M., Kieweler, R., Valenta, C., 2015. Simultaneous determination of active component and vehicle penetration from FDPPC liposomes into porcine skin layers. Eur. J. Pharm. Biopharm. 97, 90-95. DOI: 10.1016/j.ejpb.2015.10.008

Manconi, M., Caddeo, C., Sinico, C., Valenti, D., Mostallino, M.C., Biggio, G., Fadda, A.M., 2011. Ex vivo skin delivery of diclofenac by transcutol containing liposomes and suggested mechanism of vesicle-skin interaction. Eur. J. Pharm. Biopharm. 78, 27-35. DOI: $\underline{10.1016 / \text { j.ejpb.2010.12.010 }}$ 
percutaneous penetration of lipophilic materials. Int. J. Pharm. 84, 93105. https://doi.org/10.1016/0378-5173(92)90050-C

Touitou, E., Dayan, N., Bergelson, L., Godin, B., Eliaz, M., 2000. Ethosomes - novel vesicular carriers for enhanced delivery: characterization and skin penetration properties. J. Control. Release. 65, 403-418. PMID: 10699298

Valjakka-Koskela, R., Kirjavainen, M., Monkkonen, J., Urtti, A., Kiesvaara, J., 1998. Enhancement of percutaneous absorption of naproxen by phospholipids. Int. J. Pharm. 175, 225-230. https://doi.org/10.1016/S0378-5173(98)00285-3

Verma, D.D., Verma, S., Blume, G., Fahr, A., 2003a. Particle size of liposomes influences dermal delivery of substances into skin. Int. J. Pharm. 258, 141-151. PMID: 12753761

Verma, D.D., Verma, S., Blume, G., Fahr, A., 2003b. Liposomes increase skin penetration of entrapped and non-entrapped hydrophilic substances into human skin: a skin penetration and confocal laser scanning microscopy study. Eur. J. Pharm. Biopharm. 55, 271-277. PMID: 12754000

540 Yokomizo, Y., 1996. Effects of phospholipids on the percutaneous penetration of drugs through the dorsal skin of the guinea pig, in vitro. 3. The effects of phospholipids on several drugs having different polarities. J. Control. Release. 42, 217-228. https://doi.org/10.1016/0168$\underline{3659(96) 01347-8}$

Yokomizo, Y., Sagitani, H., 1996. Effects of phospholipids on the percutaneous penetration of indomethacin through the dorsal skin of guinea pigs in vitro. J. Control. Release. 38, 267274. https://doi.org/10.1016/0168-3659(95)00127-1

Yu, H.Y., Liao, H.M., 1996. Triamcinolone permeation from different liposome formulations through rat skin in vitro. Int. J. Pharm. 127, 1-7. https://doi.org/10.1016/0378$\underline{5173(95) 04055-2}$

550 Zellmer, S., Pfeil, W., Lasch, J., 1995. Interaction of phosphatidylcholine liposomes with the 
human stratum corneum. Biochim. Biophys. Acta. 1273, 176-182. PMID: 7632 


\section{Figure captions}

Fig. 1. Schematic representation of currently designed diffusion cell array system

Fig. 2. ER of skin permeation of caffeine from different phospholipid dispersions. Each value represents the mean \pm S.E. $(n=3-4) .{ }^{*}: p<0.05$ significantly different from control (free caffeine solution in PBS).

Fig. 3. Time course of the cumulative amount of caffeine that permeated through skin from different liposome formulations. Each value represents the mean \pm S.E. $(n=3-5)$. *: $p<0.05$ significantly different from control (free caffeine solution in PBS).

Fig. 4. Comparison of $E R$ of skin permeation of caffeine from phospholipid dispersions and liposomes. Each value represents the mean \pm S.E. $(n=3-5) . *: p<0.05$ significantly different for liposomes from their phospholipid dispersion.

Fig. 5. Effect of 1 h-pretreatment with caffeine-free phospholipid dispersions and liposomes on the $E R$ of skin permeation of caffeine. Each value represents the mean \pm S.E. $(n=3-5)$.

$565 *$ : $\mathrm{p}<0.05$ significantly different from control (no pretreatment; free caffeine solution in PBS).

Fig. 6. Time course of the cumulative amount of caffeine that permeated through skin from physical mixture of blank liposomes and caffeine solution (caffeine-spiked DPPG, DLPC and DSPG liposomes). Each value represents the mean \pm S.E. $(n=3-5) . *$ : $p<0.05$ significantly different from control (free caffeine solution in PBS).

Fig. 7. Time course of the normalized cumulative amount of caffeine that permeated through 
skin from caffeine-entrapped DPPG, DLPC and DSPG liposomes. Each value represents the mean \pm S.E. $(\mathrm{n}=3-5) . *$ : $\mathrm{p}<0.05$ significantly different from control (free caffeine solution in PBS). Y-axis was calculated with dividing the cumulative amount of caffeine that permeated through skin by the total amount of applied drug. 
Table 1 Abbreviation and number of carbon atom and double bond in the alkyl chain for the phopholipids used in the present study

\begin{tabular}{|c|c|c|}
\hline Full name & Abbreviation & $\begin{array}{l}\text { Carbon length : } \\
\text { double bond }\end{array}$ \\
\hline 1,2-dilauroyl-sn-glycero-3-phosphocholine & DLPC & 12:0 \\
\hline 1,2-Dilauroyl-sn-glycero-3-phosphoglycerol, sodium salt & DLPG & 12:0 \\
\hline 1,2-dimyristoyl-sn-glycero-3-phosphocholine & DMPC & 14:0 \\
\hline 1,2-dimyristoyl-sn-glycero-3-phosphoglycerol, sodium salt & DMPG & 14:0 \\
\hline 1,2-dipalmitoyl-sn-glycero-3-phosphocholine & DPPC & $16: 0$ \\
\hline 1,2-dipalmitoyl-sn-glycero-3-phosphoglycerol, sodium salt & DPPG & 16:0 \\
\hline 1,2-distearoyl-sn-glycero-3-phosphocholine & DSPC & 18:0 \\
\hline 1,2-distearoyl-sn-glycero-3-phosphoglycerol, sodium salt & DSPG & 18:0 \\
\hline 1,2-dioleoyl-sn-glycero-3-phosphocholine & DOPC & $18: 1$ \\
\hline 1,2-dioleoyl-sn-glycero-3-phosphoglycerol, sodium salt & DOPG & $18: 1$ \\
\hline
\end{tabular}


Table 2 Physicochemical properties of liposomes prepared in the present study

\begin{tabular}{lcccccc}
\hline Main component & $\begin{array}{c}T_{m} \\
\left({ }^{\circ} \mathrm{C}\right)\end{array}$ & $\begin{array}{c}E E \\
(\%)\end{array}$ & $\begin{array}{c}\text { Particle diameter } \\
(\mathrm{nm})\end{array}$ & $\begin{array}{c}\text { Polydispersity } \\
\text { Index }\end{array}$ & $\begin{array}{c}\text { Zeta } \\
\text { potential } \\
(\mathrm{mV})\end{array}$ \\
\hline DLPC & $(12: 0)$ & 49.8 & $15.44 \pm 0.74$ & $161.2 \pm 0.3$ & $0.211 \pm 0.023$ & $0.2 \pm 0.5$ \\
DLPG & $(12: 0)$ & - & $23.98 \pm 0.42$ & $109.3 \pm 0.3$ & $0.088 \pm 0.016$ & $-41.2 \pm 3.0$ \\
DMPC & $(14: 0)$ & 49.0 & $18.68 \pm 0.54$ & $165.3 \pm 2.0$ & $0.111 \pm 0.079$ & $-1.3 \pm 1.6$ \\
DMPG & $(14: 0)$ & 65.3 & $27.95 \pm 1.10$ & $130.2 \pm 0.8$ & $0.059 \pm 0.011$ & $-40.3 \pm 3.5$ \\
DPPC & $(16: 0)$ & 47.6 & $17.46 \pm 0.63$ & $169.9 \pm 2.4$ & $0.250 \pm 0.011$ & $-1.3 \pm 0.7$ \\
DPPG & $(16: 0)$ & 41.5 & $13.35 \pm 0.03$ & $152.1 \pm 0.9$ & $0.164 \pm 0.016$ & $-44.3 \pm 2.5$ \\
DSPC & $(18: 0)$ & 51.7 & $12.93 \pm 0.15$ & $184.6 \pm 3.4$ & $0.214 \pm 0.005$ & $-0.5 \pm 1.2$ \\
DSPG & $(18: 0)$ & 62.8 & $21.14 \pm 0.27$ & $162.1 \pm 0.9$ & $0.096 \pm 0.007$ & $-45.1 \pm 1.0$ \\
DOPC & $(18: 1)$ & 65.8 & $49.08 \pm 1.27$ & $143.3 \pm 1.0$ & $0.070 \pm 0.007$ & $-6.6 \pm 1.2$ \\
DOPG & $(18: 1)$ & 54.5 & $45.38 \pm 0.11$ & $110.7 \pm 0.5$ & $0.116 \pm 0.122$ & $-41.6 \pm 1.7$ \\
\hline
\end{tabular}


Fig. 1.
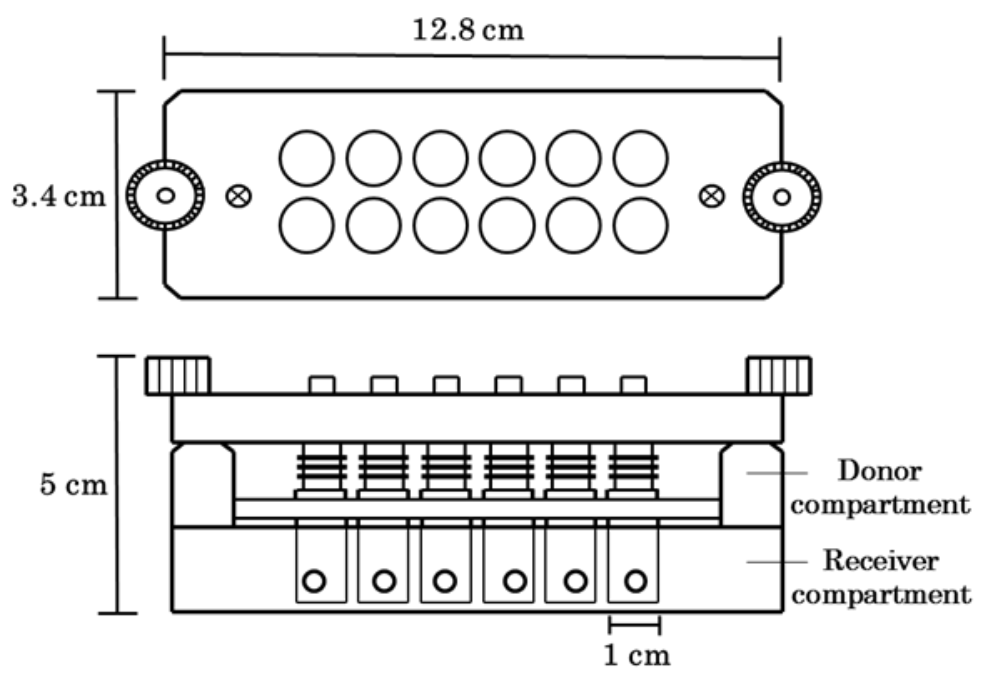
Fig. 2.

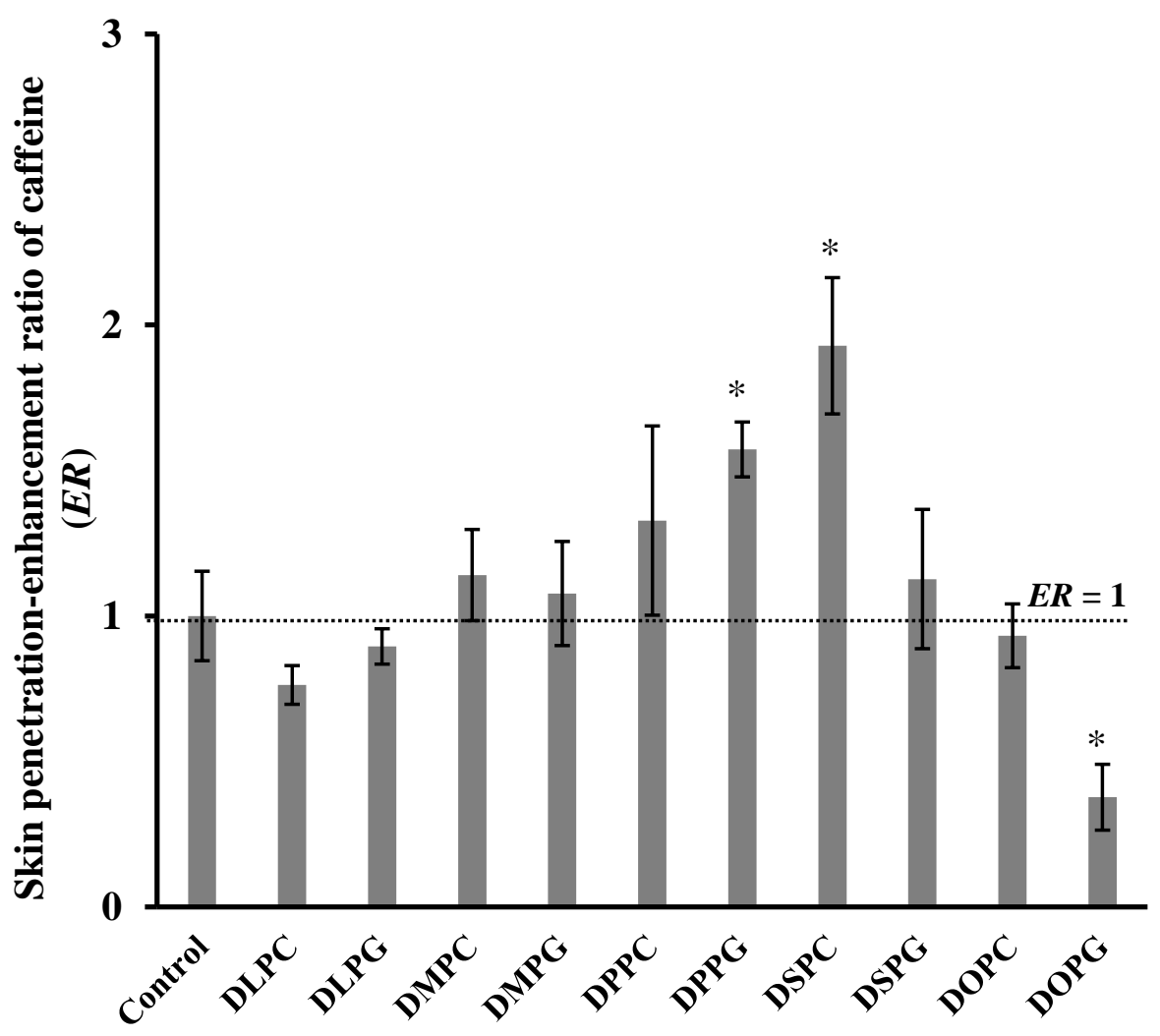


Fig. 3.

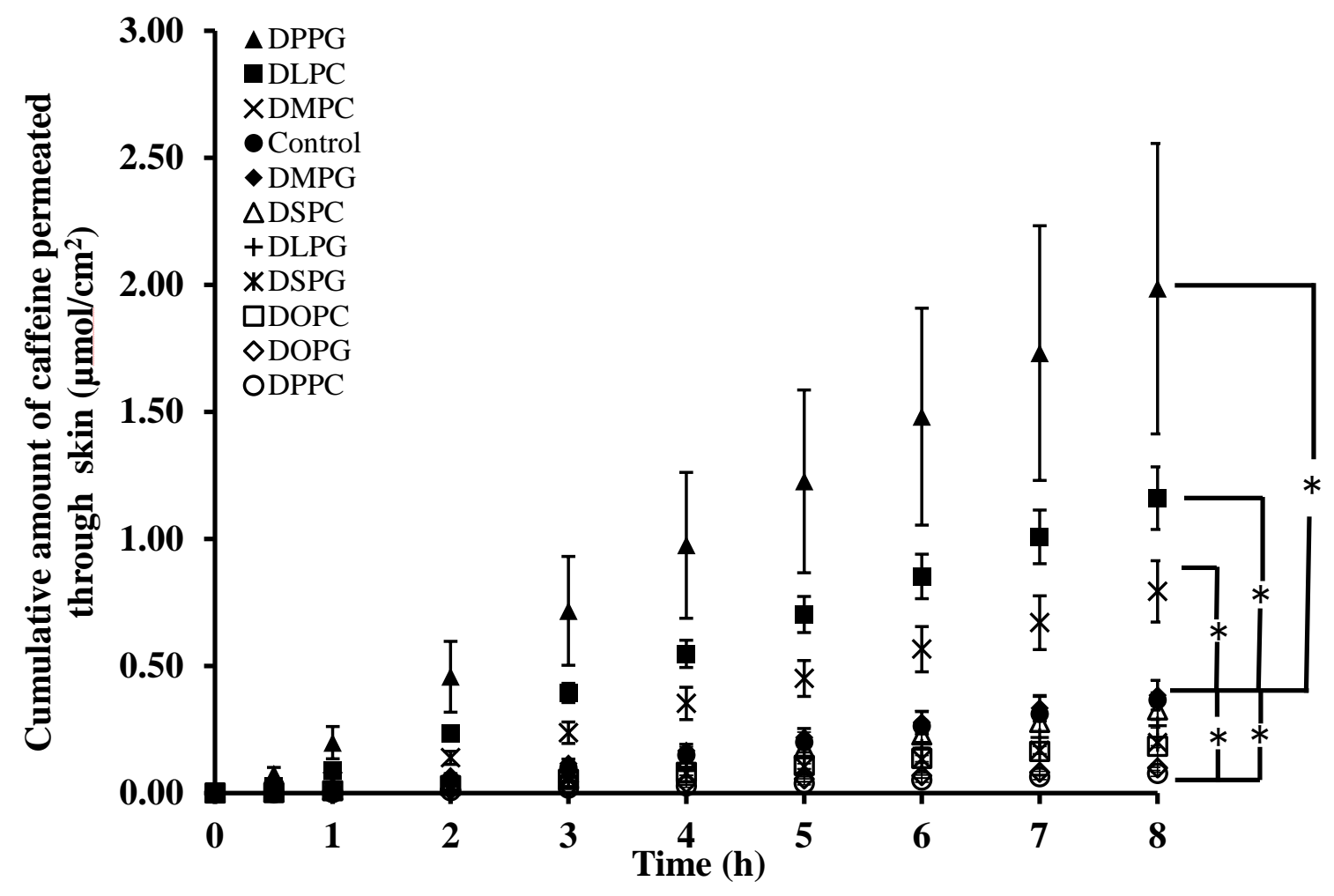




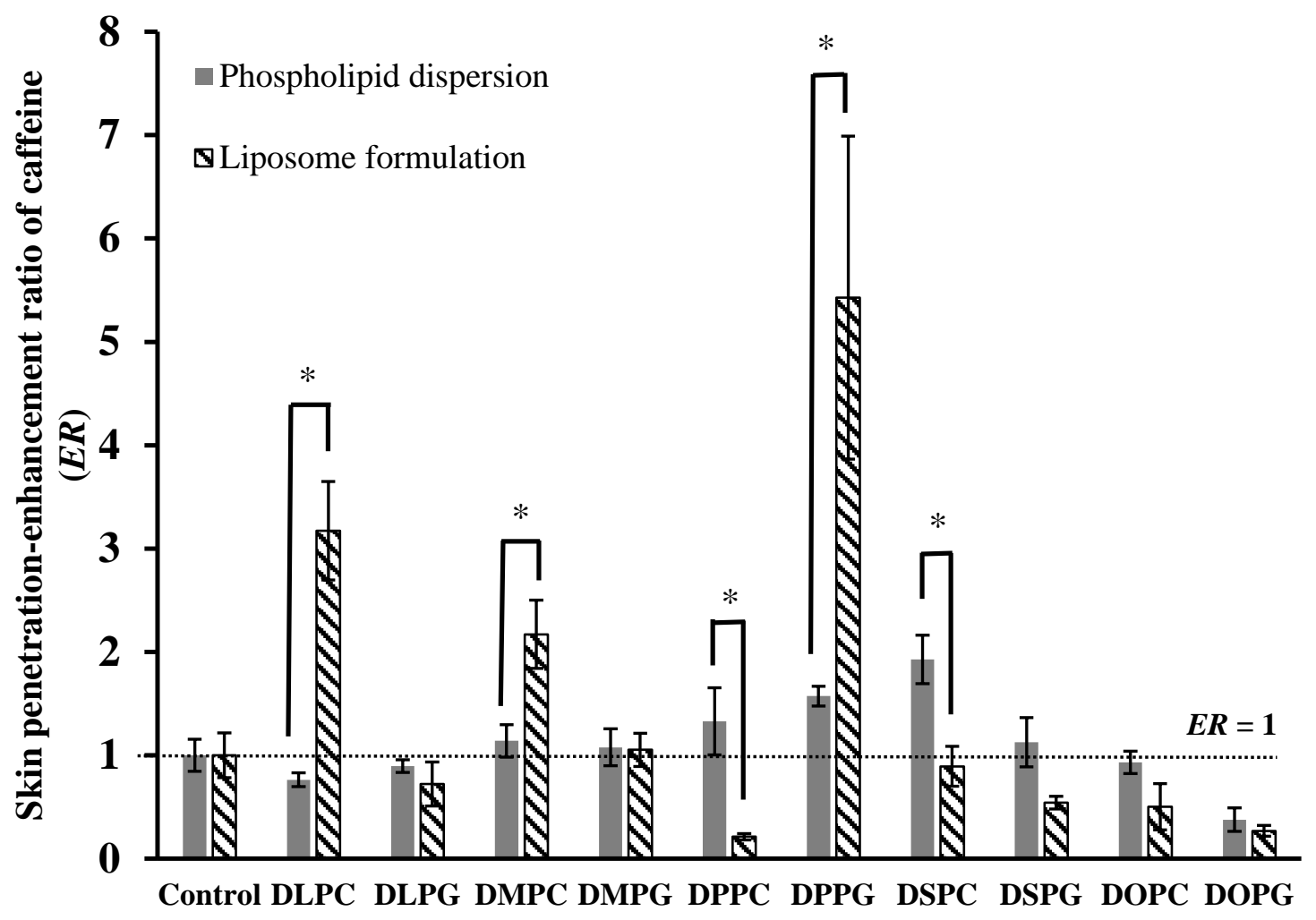


Fig. 5.

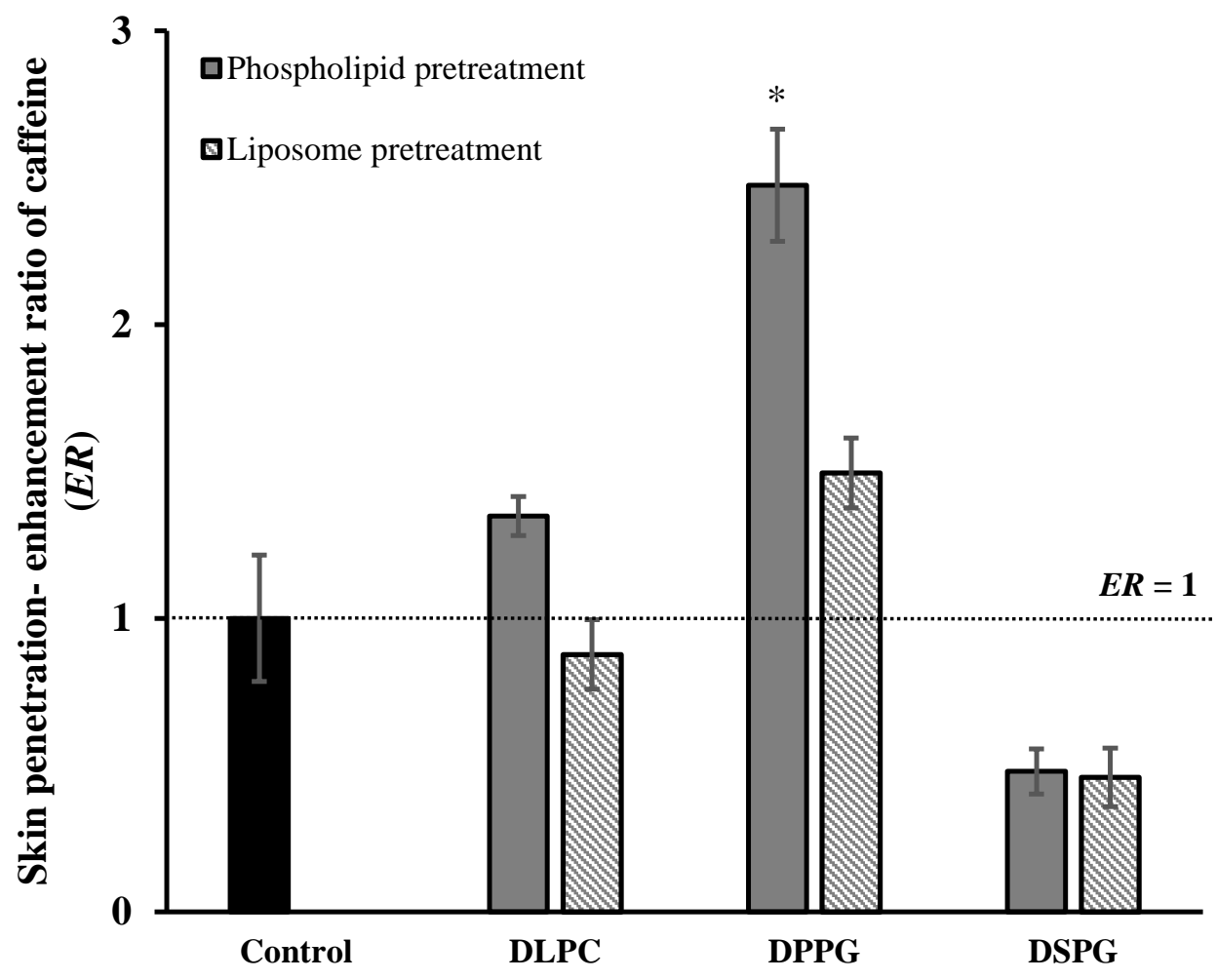

605 
Fig. 6.

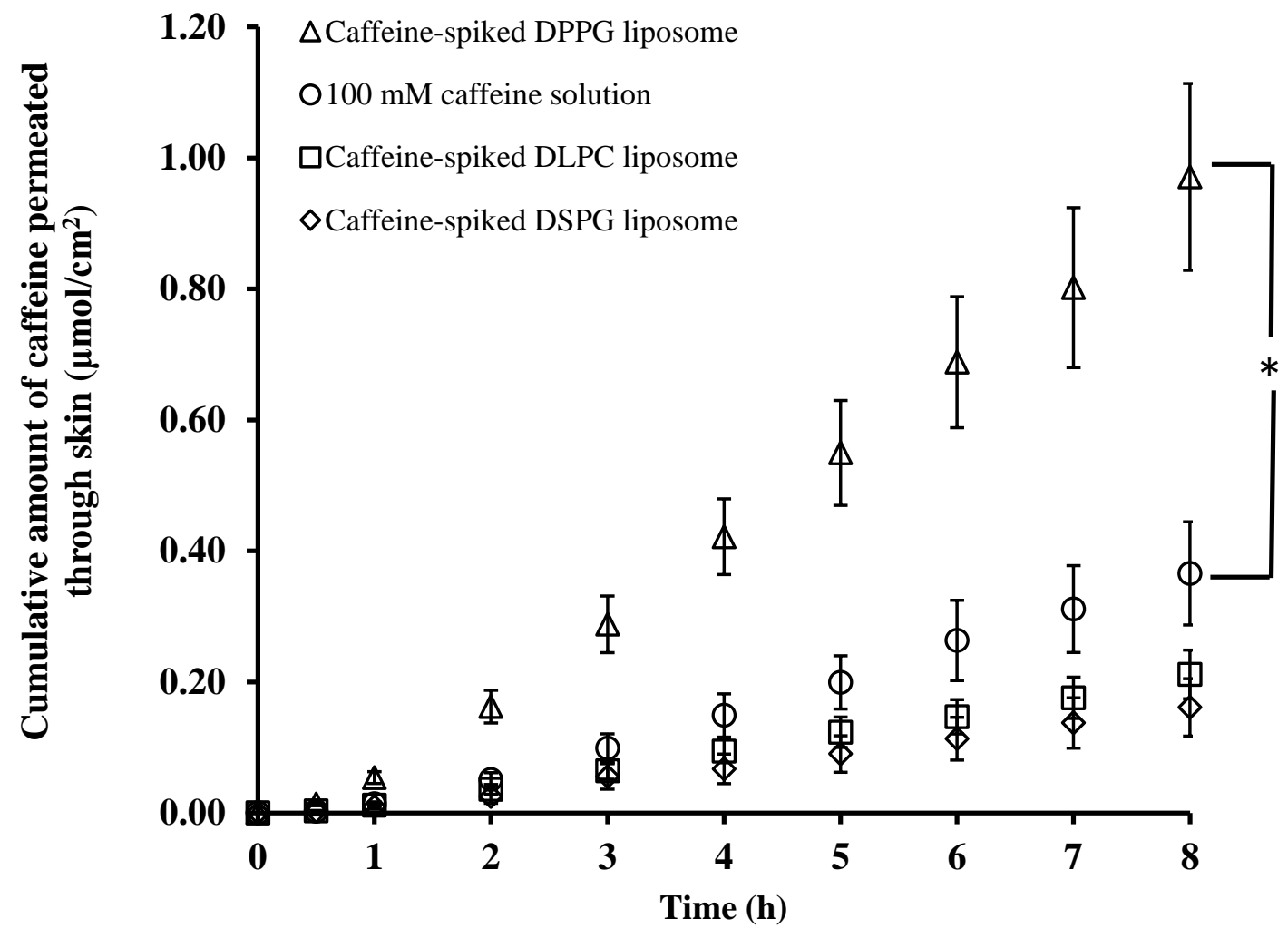


Fig. 7.

620

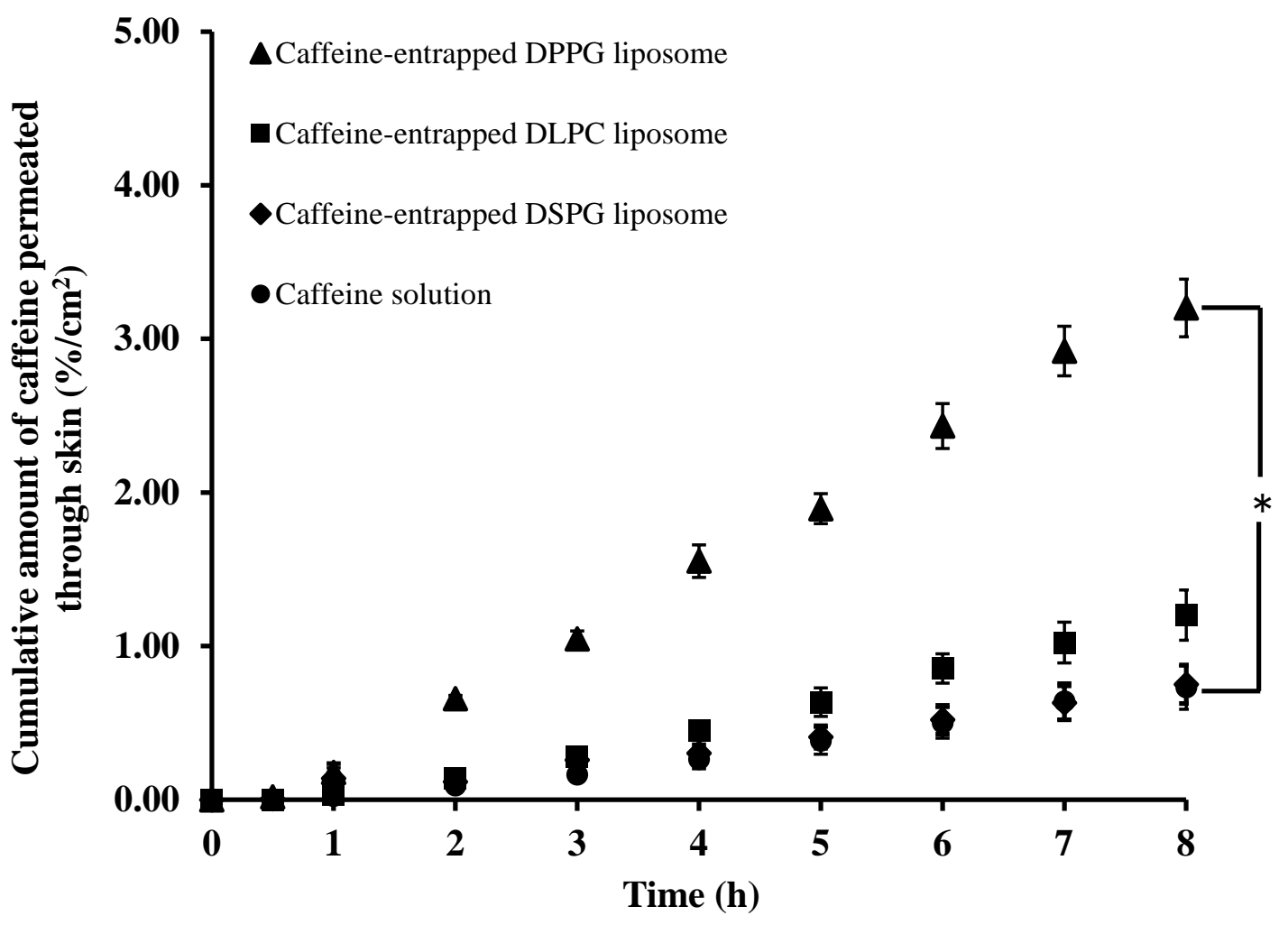

